Brit. Heart J., 1964, 26, 760.

\title{
THE PATTERNS OF PULMONARY CIRCULATION IN PULMONARY ATRESIA
}

\author{
BY \\ A. W. VENABLES \\ From the Royal Children's Hospital, Melbourne, Australia \\ Received October 28, 1963
}

Pulmonary atresia implies complete organic obstruction to normal direct outflow from the right ventricle to the pulmonary trunk. When such obstruction is present, survival is dependent on diversion of the systemic venous return to the left side of the heart through the atrial or the ventricular septum with subsequent flow of blood to the lungs through a persistent patent ductus or through bronchial or other anomalous vessels arising from the aorta. In some subjects who appear on clinical and even investigatory findings to have pulmonary atresia, necropsy reveals instead extreme pulmonary stenosis, but such an error is not very important or relevant.

This discussion presents the spectrum of phenomena associated with the varied forms of the pulmonary circulation in pulmonary atresia illustrated by specific cases, without detailing complete experience of the various syndromes, as it is felt desirable that these syndromes should be placed in perspective to each other. The development of left ventricular failure secondary to excessive pulmonary flow in some infants with pulmonary atresia and ventricular septal defect will be described. Further, attention is drawn to the probable role of a small tricuspid orifice or actual tricuspid valve stenosis in determining the size of the right ventricular cavity in patients with pulmonary atresia with an intact ventricular septum.

Pulmonary atresia is not rare. Keith, Rowe, and Vlad (1958) recorded that the incidence of pulmonary atresia was about 20 per cent in various necropsy series of the tetrad of Fallot, and referred to 46 reported cases of pulmonary atresia without ventricular septal defect proven by postmortem examination. Necropsy figures probably overestimate the incidence in the tetrad of Fallot, but the number of reported cases without ventricular septal defect is steadily rising.

The atresia may be localized to the level of the pulmonary valve with or without the presence of fused but otherwise fairly normally formed cusps, or it may be more extensive representing the ultimate severity of the usual fairly diffuse right ventricular outflow obstruction of the tetrad of Fallot. As in incomplete forms of obstruction to outflow (pulmonary stenosis); an intact ventricular septum is usually associated with localized valvar obstruction. The 6 cases recorded by Allanby et al. (1950) provide an excellent illustration of the various types of obstruction seen.

The physiological variations and clinical syndromes encountered with pulmonary atresia depend finally not on the anatomy of the atresia but on the presence or absence of a significant ventricular septal defect and on the amount and route of pulmonary flow.

\section{Pulmonary Atresia with Significant Ventricular Septal Defect}

The combination of pulmonary atresia with significant ventricular septal defect represents essentially the ultimate severity of the tetrad of Fallot, but the nature and amount of pulmonary flow 
place some of these patients in a special category. If the ventricular septal defect is small and hæmodynamically insignificant, it is best disregarded, as in the patient with a defect of $1 \mathrm{~mm}$. diameter described by Elliott, Adams, and Edwards (1963). Ordinarily the defect is of substantial size as in the usual cases of the tetrad of Fallot, with the result that right ventricular systolic pressure is adjusted to left ventricular and aortic pressures, as in the latter condition. The high right ventricular systolic pressures that may occur in isolated severe obstruction do not occur, and right ventricular failure does not develop. The left ventricular load depends on the amount of pulmonary flow, which varies considerably from patient to patient according to the size of bronchial and other anomalous collateral vessels present.

\section{(1) With Reduced Pulmonary Flow}

It is recognized that in the tetrad of Fallot bronchial flow is commonly increased without reaching sufficient quantity to affect arterial oxygen concentration significantly. Campbell and Deuchar (1961) found continuous murmurs attributed to bronchial vessels in less than 1 per cent of their cases of the tetrad of Fallot: these murmurs were usually faint and inconstant. In certain cases of pulmonary atresia with ventricular septal defect, pulmonary flow is dependent on a patent ductus, bronchial flow being similarly relatively small and unimportant to survival. Under these circumstances pulmonary flow is ordinarily reduced well below normal levels, and the aortic mixture of systemic venous and pulmonary venous blood consequently has a low oxygen saturation. These patients have considerable cyanosis from early infancy, with substantial disability. There may or may not be a continuous murmur related to flow through the ductus, but, as with other cases of pulmonary atresia, absence of direct flow from right ventricle to pulmonary trunk means that there is no pulmonary ejection murmur of the type heard in the tetrad of Fallot. There may be an early systolic click as described by Allanby et al. (1950) and by Soulié et al. (1950), though neither of these papers indicated its origin. The radiograph shows evidence of decreased pulmonary flow, with small pulmonary arteries or at times evidence of abnormal pulmonary vessels. The aortic arch may be on the right side. The heart is not significantly enlarged.

These patients may truly be described as the extreme tetrad of Fallot or "hyper-Fallot" of Soulié et al. (1950), merging as they do with patients with very severe outflow tract stenosis. Differentiation from the latter may be very difficult and perhaps unnecessary. Patients with very severe stenosis may have no ejection murmurs, and on clinical evidence may appear to have pulmonary atresia. The presence of an outflow tract may only be demonstrated by chance passage of a catheter through the obstruction or by very careful selective angiography. Venous angiocardiography, on which many diagnoses have been based in the past, is generally unsatisfactory for accurate assessment of the anatomy of such patients. The value of demonstrating the presence of severe stenosis lies in visualization of the pulmonary arteries as a guide to palliative anastomotic surgery, rendering thoracotomy less of an exploratory procedure. Physiologically the two situations are very similar. Direct reparative surgery is at present not feasible in patients with pulmonary atresia or in patients with severe stenosis unless the latter is by chance localized, at valvar or infundibular level.

In patients with pulmonary atresia and decreased pulmonary flow, life is often precariously dependent on continuing duct patency. Delayed closure of the ductus frequently occurs in infancy with increase in hypoxia heralded by increasing cyanosis and dyspnca and by the onset of restlessness leading fairly quickly to death, but prompt shunt surgery may be life saving. The technical difficulties of performing shunt operations in early infancy justify waiting for growth provided the possibility of such deterioration is anticipated, as the duct may remain open indefinitely permitting survival though usually with substantial disability.

The problems of assessment are illustrated by the following case reports.

Case 1. A baby boy was cyanosed from birth. At 22 months he exhibited a præcordial systolic murmur with a continuous murmur in the left scapular region. A venous angiogram showed a ventricular septal 
defect with a single right-sided arterial trunk from which the pulmonary arteries appeared to fill. The condition was diagnosed as pulmonary atresia.

When reviewed at 11 years he was deeply cyanosed with severe disability. There was an ejection sound at the apex. No murmurs were audible over the heart, but soft continuous murmurs were present on both sides posteriorly. Selective angiography from the right ventricle demonstrated a narrow outflow tract leading to small pulmonary arteries. The right pulmonary artery, which measured $6 \mathrm{~mm}$. in diameter, was subsequently mobilized and anastomosed directly to the aorta with substantial improvement in his colour.

Case 2. A baby boy was seen at the age of 9 days with cyanosis and evidence of cerebral birth injury. There was then virtually no murmur, but a continuous murmur which had previously been heard at the pulmonary area subsequently reappeared. Oxygen saturation was 73 per cent at rest. The cardiogram showed normal right ventricular activity, while radiography revealed a normal-sized heart with a concave pulmonary segment and dry lung fields. He recovered from the cerebral lesion with little evidence of residual damage, and at 6 months investigation revealed evidence of a severe form of the tetrad of Fallot with a very narrow right ventricular outflow tract with a small pulmonary artery at least partly filled through a ductus. At 7 months there was progressive deterioration over several weeks, with evidence of increasing hypoxia. Aorto-pulmonary anastomosis was performed successfully.

The only previous child of this mother, by a different husband, died earlier at the age of 6 years from cerebral abscess complicating ventricular septal defect with pulmonary vascular obstruction.

The occurrence of certain more complex anomalies in combination with pulmonary atresia and free interventricular communication may be of surprisingly little physiological importance. Such conditions include mitral atresia and anomalies of systemic venous drainage. In such patients mixing of pulmonary and systemic venous blood, which ordinarily occurs at aortic level, merely occurs earlier, and the degree of arterial desaturation is controlled by the same factors. Ventricular loading is the same as in uncomplicated pulmonary atresia with ventricular septal defect.

Differential diagnosis of pulmonary atresia with reduced pulmonary flow includes other cyanotic anomalies with dry lung fields and relatively small hearts, such as certain cases of tricuspid atresia.

\section{(2) With Increased Pulmonary Flow}

In some patients with pulmonary atresia there may be considerable flow to the lungs through bronchial or anomalous collateral arteries. These vessels may anastomose directly with the pulmonary arteries in the hilum (Allanby et al., 1950; Soulié et al., 1950; and Taussig, 1960) and may in fact provide a wide vascular ring between the aorta and a main pulmonary artery of relatively normal size beyond the atresia (Fig. 1). Such major central anastomoses between systemic vessels and the pulmonary artery branches are regarded as the source of the characteristic "bronchial" continuous murmurs, recognition of this being attributed to Routier by Soulié et al. (1950). In Case 3 where there was an actual direct ring a systolic murmur only was heard. Lesser direct anastomoses may also exist without audible continuous murmurs (Allanby et al., 1950; Campbell and Deuchar, 1961).

The origin of the systemic arterial supply to the lungs has been discussed fully by Allanby et al (1950). Relation to possible absence of the ductus is not clear in what has been written. Powell and Hiller (1957) suggested that the systemic-pulmonary anastomoses may develop in fœtal life in association with pulmonary atresia because of hypertrophy of collateral supply secondary to lack of formation of the ductus. It seems at least equally likely that pulmonary atresia is accompanied in some patients by further developmental abnormality of the alternative pulmonary supply in which the ductus is actually replaced at times by other aorto-pulmonary communications of similar origin. Such a situation was seen in its most complete form in the current Case 3 (Fig. 1). It seems almost certain that direct bronchopulmonary communications may also be present with evidence of a closed ductus or ligamentum arteriosum.

Case 3. A baby boy was admitted at 1 month with micrognathia and failure to thrive. He appeared dusky, his arterial saturation being 85 per cent. There was a basal systolic murmur loudest at the aortic area. Radiography showed slight cardiac enlargement with moderate pulmonary plethora. The cardio- 


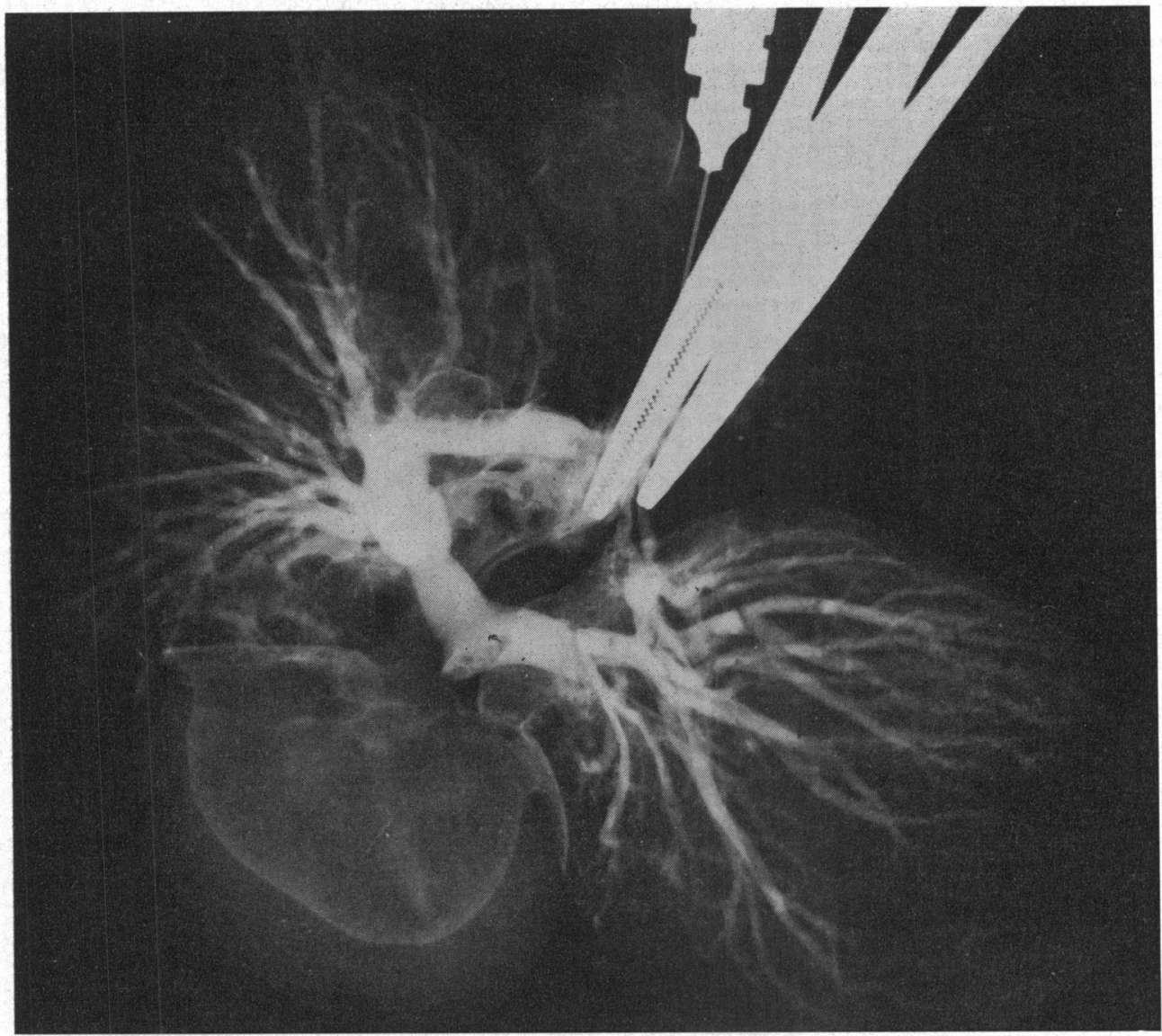

FIG. 1.-Post-mortem specimen of pulmonary atresia. Radiograph following injection of radioopaque dye into aorta between clamps. (See text.)

gram showed a right ventricular pattern with an upright T in V1. Progress was poor with continuing respiratory distress and episodic deterioration with chest signs attributed to pulmonary infection and heart failure. At $2 \frac{1}{2}$ months cardiac investigation revealed the presence of pulmonary atresia with ventricular septal defect and bronchial arterial supply to the lungs. He died at $5 \frac{1}{2}$ months. Necropsy confirmed the investigatory findings. Arteries from the descending aorta anastomosed freely with the pulmonary arteries on both sides anteriorly to form an arterial ring from which pulmonary branches arose (Fig. 1).

Pulmonary flow may be increased beyond normal in patients of this type, with the result that the arterial oxygen saturation is relatively high and symptoms are minimal. Such a clinical situation has been described by Soulié et al. (1950), Powell and Hiller (1957), Campbell and Deuchar (1961), and others. In stressing the occurrence in some patients of increased pulmonary flow with mild cyanosis and only moderate disability, Campbell and Deuchar emphasized the difficulties of being sure of the exact anatomical diagnosis in the "pseudo-truncus" group, and classified these cases into groups of persistent truncus arteriosus, "solitary trunk", and proven pulmonary atresia though almost certainly the majority of the "solitary trunk" group would actually have been examples of pulmonary atresia.

The magnitude of the increase in pulmonary flow may be assessed by calculation of the ratio of pulmonary to systemic flow from investigatory findings if available, assuming that there is complete 
mixing of pulmonary and systemic venous blood in the aorta and that the lungs are supplied solely with systemic arterial blood. Thus,

$$
\frac{\text { Pulmonary flow }}{\text { Systemic flow }}=\frac{\text { Aortic saturation }- \text { mixed systemic venous saturation }}{\text { Pulmonary artery saturation-pulmonary venous saturation }} \text {. }
$$

In these cases aortic saturation may be substituted for pulmonary artery saturation. Pulmonary venous saturation may need to be assumed. In the 9 patients of Campbell and Deuchar (1961) labelled as "solitary trunk" with increased pulmonary flow, the measured pulmonary flows were approximately twice normal and arterial oxygen saturations averaged 83 per cent, the highest being 89 per cent. Of 18 patients labelled purely as pulmonary atresia, 2 had little disability. The left ventricle usually tolerates well the increase in stroke output which such increased pulmonary flow entails, and the prognosis is excellent because of stability of the hæmodynamic situation. Campbell and Deuchar (1961) record survival without deterioration for an observed interval of 13 years to the age of 30 years at the time of their report. Bacterial endocarditis and cerebral abscess are both complications that may occur with lethal or crippling effects. Case 6 of the report of Soulié et al. (1950) died at 18 years from bacterial endocarditis, while the following case report illustrates the problem of cerebral abscess. As in other subjects with right-to-left shunt, the possibility of development of cerebral abscess must be kept in mind constantly whenever appropriate symptoms develop.

Case 4. A baby boy was found in infancy to have cyanosis and bilateral continuous murmurs. A venous angiocardiogram at the age of 3 years was interpreted as showing a "pseudo-truncus" with a right aortic arch and bronchial flow. Review of the films shows a narrow right ventricular outflow tract with filling of a small infundibular chamber. His colour remains good and he has only mild clubbing. At 11 years he presented with a five-day history of increasing headache with subsequent development of coma and hemiparesis, due to a large right-sided cerebral abscess from which no organisms were grown. He recovered following neurosurgical intervention with only minor residual defect.

An operation is not indicated in patients with the type of pulmonary atresia with increased pulmonary flow. Such patients in fact stand to lose from exploratory thoracotomy.

The condition is diagnosed from the association of mild to moderate cyanosis and clubbing with continuous murmurs over the lung fields and typical radiological findings. There is usually a deep angular bay in the pulmonary artery segment. The normal pulmonary arterial pattern is replaced by that of bronchial supply described by Campbell and Gardner (1950). The aorta is large and its arch may be right-sided as in the tetrad of Fallot and in other cases of pulmonary atresia with ventricular septal defect. Selective right ventricular angiocardiography should confirm the diagnosis. As implied by Campbell and Deuchar's classification, it may not be possible during life to achieve satisfactory differentiation from the uncommon form of persistent truncus arteriosus in which the lungs are supplied only by vessels arising from the descending aorta.

Transitional forms undoubtedly exist with pulmonary flow of more normal dimensions and correspondingly greater disability. In addition there are some subjects in whom pulmonary flow is so great that left ventricular failure occurs in early infancy (Case 5, below). In the older, stable subjects the left ventricular load either always has been tolerated well, or has become tolerable by decrease as illustrated in Case 5. In Case 3, left ventricular failure of similar origin was also probably an important factor in the outcome. Rather similar disturbances in left ventricular function occur following a systemic-pulmonary anastomosis that leads to excessive pulmonary flow. In the latter patients left ventricular failure may be precipitated simply by the volume load, or may be potentiated by other factors such as relative obstruction to left ventricular outflow occurring in a patient with double outlet right ventricle and pulmonary stenosis following a shunt operation. In patients with pulmonary atresia and congenital increase in pulmonary flow, the magnitude of the flow is the factor that precipitates failure. 
Case 5. A baby girl was admitted at the age of 13 days for investigation of apparent genital abnormality. Two days later she became dyspnoic and appeared to be in heart failure. She was dusky, and ear oximetry gave readings of 84 per cent saturation in air and 95 per cent in oxygen. Her pulses were jerky and there were loud murmurs which initially appeared to be continuous to the left of the sternum but only systolic to the right of the sternum, but later appeared to be continuous in all areas. Radiography showed a right aortic arch with opacity in the right upper lung zone due to abnormal vasculature. The cardiogram suggested abnormal left ventricular preponderance. She remained very ill and dyspnœic, and thoracotomy was performed at $\mathbf{1 6}$ days. A large systemic vessel entering the right pulmonary artery was ligated. This is considered to have been a major bronchopulmonary communication, not a right-sided ductus as was thought at the time of operation. Subsequent selective angiocardiography revealed the presence of a large single right-sided arterial trunk with bronchial vessels entering the right lung. There was no immediate change, but over the 18 months since admission there has been gradual over-all improvement with some increase in cyanosis. The widespread continuous murmurs persist. In this patient pulmonary flow was sufficient to produce peripheral manifestations of the aortic run-off. Recovery may well be due to increasing pulmonary vascular resistance.

\section{Pulmonary Atresia with InTact Ventricular SePtum}

Pulmonary atresia with intact ventricular septum has synonyms, such as pulmonary atresia with normal aortic root and isolated pulmonary atresia, which correspond exactly to the nomenclature of pulmonary stenosis without ventricular septal defect.

The atresia is frequently valvar with an imperforate valve membrane showing triradiate ridges corresponding to valve commissures. The pulmonary trunk is usually somewhat small, and is described by Davignon et al. (1961b) as having a funnel-shaped appearance where it arises from the valve site. In the patient of Allanby et al. (1950) who had this condition, the pulmonary trunk was, however, huge and aneurysmal. The occurrence of membranous valvar obstruction and the variations in size of the pulmonary trunk are of considerable surgical importance, as are the features of the right ventricle and tricuspid valve to be discussed. The cases described have been classified into two types depending on the size of the right ventricular cavity. In the first, the right ventricular cavity is small though the wall is thickened, and in the other type the ventricular cavity is normal or large. Among the 20 cases of Davignon et al. (1961b), there were 13 with a small or tiny right ventricular cavity, the corresponding figures in the report of Benton et al. (1962) being 6 out of 12 and in the report of Elliott et al. (1963) being 9 out of 12. Thus only approximately one-third of the total cases recorded by these authors had a normal or large right ventricular cavity. Abnormal communications between the right ventricular cavity and the coronary arteries occur in the first group.

Tricuspid valve anomalies, which often consist of an incomplete variety of Ebstein's anomaly, are frequently observed and have been regarded as playing an important role in determination of the size of the right ventricular cavity through the causation of organic tricuspid regurgitation. This relation was suggested by Edwards (1960). Elliott et al. (1963) describe the presence of mild Ebstein type abnormalities of the tricuspid valve in 7 of their 9 patients with a small right ventricular cavity, but suggest that the valves were nevertheless probably competent. Such lesions had previously been described only in cases with a normal or large ventricular cavity. Of these 9 cases, however, 7 had systolic murmurs which by description appear compatible with tricuspid regurgitation. The right atrium is large and at times aneurysmal in patients with substantial tricuspid regurgitation due to abnormality of the valve.

The following case (Case 6) provides an example of gross right ventricular and right atrial dilatation in association with pulmonary atresia and abnormality of the tricuspid valve, similar to those described by Davignon et al. (1961b) and by Caddell and Whittemore (1963). Case 7 is of some interest since it appears to represent an unusual variant of the Ebstein anomaly rather than of tricuspid atresia.

Case 6. A baby boy was admitted at 2 days, severely ill with evidence of heart failure associated with greyish cyanosis. There was a loud systolic murmur and thrill over the lower sternum. Radiography 
showed gross enlargement of the heart to right and left with dry lung fields. The cardiogram showed right atrial hypertrophy with prolongation of the ventricular complexes to $0.10 \mathrm{sec}$. These findings suggested Ebstein's anomaly of the tricuspid valve, which appeared to be confirmed by cardiac catheterization, as the catheter looped widely to the left border of the heart without appearing to leave the right atrium as far as could be judged from the pressure tracings. Radiopaque dye was injected by hand into what appeared on pressures to be a small right ventricular cavity at the left border of the heart. Dye refluxed from here into the main cavity. Pressure injection into the right atrium merely confirmed the dilatation of that chamber. The baby died at the age of 1 week. Necropsy revealed gross dilatation of the right atrium and of the right ventricle, and the ventricular wall was extremely thin. The tricuspid ring was correspondingly dilated, while the tricuspid valve showed a partial Ebstein-like anomaly affecting the septal cusp. There was also pulmonary valve atresia with a small pulmonary artery. The ductus was patent, and there was an atrial septal defect. The ventricular septum was intact.

Case 7. A baby boy was admitted with cyanosis when aged 10 days. There was a triple rhythm over the præcordium but no systolic murmur was heard. Radiography showed a heart of normal size with dry lung fields. The cardiogram showed a right ventricular pattern with a $Q R$ in the right chest leads. The baby deteriorated fairly suddenly and died on the sixteenth day.

Necropsy revealed that the tricuspid valve formed an imperforate sac almost completely lining the right ventricular cavity and attached to the wall of the ventricle at several sites, with only a small slit-like space between the valve tissue and the ventricular wall. The right ventricle was somewhat small. There was atresia of the proximal $1.5 \mathrm{~cm}$. of the pulmonary artery, which was represented by a cord $1 \mathrm{~mm}$. in external diameter. Beyond this the pulmonary artery was small but patent. The ductus was patent and there was an atrial septal defect.

In isolated pulmonary atresia without ventricular septal defect, survival is dependent on flow of systemic venous blood from the right atrium to the left atrium, usually through a patent foramen ovale, and on the presence of a patent ductus: the bronchial vessels are not hypertrophied. As in those cases of pulmonary atresia with ventricular septal defect with pulmonary flow dependent on duct patency, the pulmonary flow is poor. Cyanosis is observed soon after birth and rapidly becomes severe. The expectation of life is poor, death occurring relatively early from hypoxia with or without evidence of heart failure. In contrast with the usual progress of such patients, the patient described by Allanby et al. (1950) died at 14 years following thoracotomy. Davignon et al. (1961b) describe the development of increasing cyanosis and dyspnœa which suggest decrease in flow through the ductus due to delayed closure. If the condition of isolated pulmonary atresia is recognized, pulmonary valvotomy may be curative, provided that the anatomy is compatible with establishment of an effective pulmonary circulation. Keith et al. (1958) record 3 survivals from 8 operations. In some patients a shunt procedure may be indicated.

The clinical features depend partly on the size of the right ventricle. There is no ejection murmur, but pansystolic murmurs apparently due to tricuspid regurgitation have been described regularly. There may be a triple rhythm over the præcordium. The radiograph shows ventricular enlargement corresponding in amount to the type of lesion, with considerable right atrial enlargement up to aneurysmal size in patients with significant organic tricuspid regurgitation. Heart size increases progressively in those patients of both types who survive the neonatal period, the duration of survival being similar in both groups (Davignon et al., 1961b).

The electrocardiogram is stated usually to show right axis deviation in both groups, with an early ventricular pattern which can be related to right ventricular mass. Surviving patients show evidence of increasing right atrial and right ventricular hypertrophy (Benton et al., 1962), also present in the two cases to be described. Selective angiocardiography from the right ventricle is required for final diagnosis, typical findings being illustrated by Kjellberg et al. (1959), by Davignon et al. (1961a), and by Egenberg (1963).

Case 8. A baby girl was admitted at the age of 2 days with cyanosis present since birth. There was an indeterminate systolic murmur. The radiograph showed some cardiac enlargement with dry lung fields. The cardiogram suggested left ventricular preponderance, but right axis deviation was present. Cyanosis persisted and she failed to thrive, and died at 3 months of age after the onset of heart failure. At this time 
the systolic murmur was then moderately loud and maximal over the lower sternum, and there was palpable systolic pulsation of the liver. Radiography showed that the heart had become larger, and there was evidence of right atrial, and of right ventricular hypertrophy. Necropsy revealed pulmonary valve atresia with normal cusp outlines. The tricuspid valve was normal and the right ventricle showed hypertrophy of its wall with a somewhat dilated cavity. The right atrium was dilated.

Case 9. A baby boy was admitted at the age of 1 week with cyanosis which had been first noticed on the fourth day of life. There was a systolic murmur at the left mid-sternal edge with a continuous murmur at the pulmonary area. Radiography showed a bulky heart with dry lung fields while the cardiogram showed left ventricular preponderance. The continuous murmur disappeared and he became more cyanosed, oxygen saturation being then 61 per cent at rest. At the age of 9 weeks there was further deterioration with attacks of screaming. Further radiographs showed increased cardiac enlargement, and the cardiogram now showed right atrial hypertrophy and right ventricular hypertrophy. Cardiac catheterization disclosed a right ventricular systolic pressure ranging between 75 and $125 \mathrm{~mm} . \mathrm{Hg}$, left ventricular systolic pressure being $90 \mathrm{~mm}$.

Selective angiocardiography performed from the right ventricle indicated the presence of probable pulmonary atresia without ventricular septal defect, dye leaving the right ventricle by reflux through the tricuspid valve to the right atrium. There was only doubtful evidence of direct passage of dye to the pulmonary artery in the antero-posterior view, not confirmed in the lateral view.

Transventricular valvotomy was performed, a Brock dilator being finally opened in the outflow tract to 5 to $6 \mathrm{~mm}$. The baby's colour improved and the arterial saturation two days later was 77 per cent, but general progress was unsatisfactory, with later complete breakdown of the chest wound. Death occurred four weeks after operation.

Necropsy revealed a lacerated pulmonary valve of $4 \mathrm{~mm}$. diameter with only a small effective channel. The right ventricle was hypertrophied with a somewhat small cavity showing endocardial thickening. The tricuspid valve was moderately stenosed (diameter $8 \mathrm{~mm}$.), with a buttonhole orifice. The diameter of the mitral valve was $14 \mathrm{~mm}$.

Certain aspects of this condition are still not clear, particularly the basis of the size of the right ventricular cavity. While quite clearly organic tricuspid regurgitation would increase the volume load of the right ventricle and predispose to dilatation, anomalies of the tricuspid valve are not constantly present in such cases, being apparently absent in the 3 cases of Schrire, Sutin, and Barnard (1961), in 1 patient of Davignon et al. (1961b), and in 3 patients of Benton et al. (1962) placed within the category of normal right ventricular cavity size. Right ventricular hypertension has been demonstrated in the presence of a small right ventricular cavity (Davignon et al., 1961a), and may be deduced to be present regularly from the observed right ventricular hypertrophy. The electrocardiographic changes in surviving patients further indicate that this hypertrophy is progressive. The onset of failure as a consequence of the right ventricular loading is quite acceptable. Tricuspid regurgitation may of course occur as a phenomenon secondary to right ventricular failure as in severe pulmonary valve stenosis, but is then truly "functional".

There is considerable similarity between isolated extreme pulmonary valve stenosis and isolated pulmonary atresia, as demonstrated by Benton et al. (1962). Of their 17 patients defined as extreme pulmonary valve stenosis on a basis of a measured pulmonary valve orifice of $1.5 \mathrm{~mm}$. diameter or less, 7 had a right ventricular cavity of less than half normal size. Developmental abnormalities of the tricuspid valve leading to the organic type of regurgitation appear to be rare in pulmonary valve stenosis, and were not described in their patients. Of the 7 patients with a small right ventricular cavity, 5 had small but otherwise normal tricuspid valves. On the other hand, tricuspid stenosis is seen from time to time in association with pulmonary valve stenosis. The papers of Davignon et al. (1961b) and Elliott et al. (1963) both refer to the small size of the tricuspid valve in the type of pulmonary atresia with a small right ventricle. Davignon et al. (1961b) in fact describe these tricuspid valves as tiny. The possibility that follows from these facts is that difficulty in right ventricular filling, due to obstruction to inflow presented by a small tricuspid orifice, may in fact prevent the onset of right ventricular dilatation while hypertrophy can still occur because of the high pressures developed in the ventricle from non-ejectile isometric contraction. A normal 
tricuspid valve would allow more effective ventricular filling with the likelihood of later progressive dilatation from heart failure, while an organically incompetent tricuspid valve would potentiate dilatation and so be likely to be associated with greater degrees of this phenomenon. This hypothesis certainly appears to merit further consideration.

Differential diagnosis of pulmonary atresia with intact ventricular septum includes tricuspid atresia with underdevelopment of the right ventricle, and Ebstein's anomaly of the tricuspid valve without pulmonary atresia. Tricuspid atresia may of course be combined with pulmonary atresia and a non-functioning right ventricle. The electrocardiogram of tricuspid atresia shows a stable pattern of left ventricular dominance with left axis deviation, in contrast with the right axis deviation seen in pulmonary atresia, which in survivors increases in association with increasing evidence of right atrial and right ventricular hypertrophy (Benton et al., 1962). Isolated Ebstein's anomaly may present in infancy with cyanosis, dry lung fields, and a cardiac contour similar to that of pulmonary atresia. The presence of a triple rhythm, in this case not due to heart failure, in the absence of an ejection murmur may further confuse the issue. Case 4 of Schrire et al. (1961), in whom there was Ebstein's anomaly with a patent ductus but a normal pulmonary valve, resembled their three cases of pulmonary atresia apart from the presence of a conduction defect in the electrocardiogram. The investigatory findings in Case 6 of this report suggested Ebstein's anomaly by virtue of the way in which the catheter looped to the left border of the heart while apparently still in the right atrium, a ventricular pattern being obtained only at the extreme left border of the heart. The explanation of the latter finding is not clear, and the correct diagnosis was not made until necropsy. This merely confirms the importance of making investigatory procedures as complete as possible. If a more adequate selective angiogram had been performed from the region where the pressure tracing suggested a small right ventricular cavity, the diagnosis might perhaps have been made in life.

\section{General Comment}

The spectrum of the pulmonary circulation in pulmonary atresia is wide. When the atresia is associated with significant ventricular septal defect the syndromes seen range from the precarious hypoxic state of the subject dependent for pulmonary circulation on a patent ductus to the relative well-being consequent on increased pulmonary flow provided by large bronchial or anomalous vessels of systemic origin. The price of high levels of arterial saturation may be left ventricular failure early in infancy if the left ventricle cannot cope with the pulmonary flow. Where there is no ventricular septal defect there is once again a precarious hypoxic state complicated in some by right ventricular failure.

The clinical problems vary, therefore, from acute problems in infancy with hypoxia and heart failure of various patterns to long-term situations with often mild or moderate disability, in which the complications of bacterial endocarditis and of cerebral abscess may arise, and special social and emotional problems must be met.

\section{SUMMARY}

The syndromes associated with pulmonary atresia are determined by the presence or absence of significant ventricular septal defect and by the extent and route of pulmonary blood flow. Patients in whom pulmonary atresia is associated with ventricular septal defect may have a small pulmonary flow precariously dependent on a patent ductus, or a larger, sometimes increased, pulmonary flow through bronchial or anomalous systemic arteries which may anastomose directly with hypoplastic pulmonary arteries. The prognosis in the latter group is usually regarded as good, but the occurrence in infancy of left ventricular failure from excessive pulmonary flow is now described.

Patients with isolated pulmonary atresia, in whom pulmonary flow is again precariously dependent on duct patency, have a poor prognosis, with severe hypoxia associated at times with right 
ventricular failure. These patients may be divided into two groups according to the size of the right ventricular cavity. The relation of organic tricuspid regurgitation to right ventricular dilatation is discussed further, and the suggestion is made that impaired ventricular filling due to a small tricuspid orifice may be of importance in patients with small right ventricular cavities.

The author is grateful to those members of the Senior Medical Staff of the Royal Children's Hospital who have referred patients for consultation and investigation. The post-mortem examinations were performed in the Department of Pathology of the Hospital.

\section{REFERENCES}

Allanby, K. D., Brinton, W. D., Campbell, M., and Gardner, F. (1950). Pulmonary atresia and the collateral circulation to the lungs. Guy's Hosp. Rep., 99, 110.

Benton, J. W., Elliott, L. P., Adams, P., Anderson, R. C., Hong, C. Y., and Lester, R. G. (1962). Pulmonary atresia and stenosis with intact ventricular septum. Amer. J. Dis. Child., 104, 161.

Caddell, J. L., and Whittemore, R. (1963). Pulmonary atresia with dilated right ventricle: a case with congenital atrial flutter. Amer. J. Cardiol., 12, 254.

Campbell, M., and Deuchar, D. C. (1961). Continuous murmurs in cyanotic congenital heart disease. Brit. Heart $J ., 23,173$.

—, and Gardner, F. (1950). Radiological features of enlarged bronchial arteries. Brit. Heart J., $12,183$.

Davignon, A. L., DuShane, J. W., Kincaid, O. W., and Swan, H. J. C. (1961a). Pulmonary atresia with intact ventricular septum: report of 20 cases studied by selective angiocardiography and right heart catheterization. Amer. Heart J., 62, 690.

—, Greenwold, W. E., DuShane, J. W., and Edwards, J. E. (1961b). Congenital pulmonary atresia with intact ventricular septum: clinicopathologic correlation of two anatomic types. Amer. Heart J., $62,591$.

Edwards, J. E. (1960). Pulmonary atresia with intact ventricular septum. In Pathology of the Heart, 2nd ed., ed. S. E. Gould, p. 397 . Thomas, Springfield, Illinois.

Egenberg, K. E. (1963). Pulmonary atresia with intact ventricular septum. Brit. Heart J., 25, 540.

Elliott, L. P., Adams, P., Jr., and Edwards, J. E. (1963). Pulmonary atresia with intact ventricular septum. Brit. Heart J., 25, 489.

Keith, J. D., Rowe, R. D., and Vlad, P. (1958). Heart Disease in Infancy and Childhood, pp. 399, 569. Macmillan, New York.

Kjellberg, S. R., Mannheimer, E., Rudhe, U., and Jonsson, B. (1959). Diagnosis of Congenital Heart Disease, 2nd ed., p. 230. The Year Book Publishers, Chicago.

Powell, M. L., and Hiller, H. G. (1957). Congenital ducto-pulmonary atresia: a variant of pulmonary atresia of some clinical importance. Med. J. Aust., 2, 684.

Schrire, V., Sutin, G. J., and Barnard, C. N. (1961). Organic and functional pulmonary atresia with intact ventricular septum. Amer. J. Cardiol., 8, 100.

Soulié, P., Nouaille, J., Schweisguth, O., and Touche, M. (1950). Le "truncus aorticus" (diagnostic clinique et radiologique). Bull. Soc. méd. Hôp. Paris, 66, 919.

Taussig, H. (1960). Congenital Malformations of the Heart, 2nd ed., Vol. 2, p. 276. The Commonwealth Fund, Harvard University Press, Cambridge, Massachusetts. 